\title{
Two-Dimensional Homogeneous Fermi Gases
}

\author{
Klaus Hueck, ${ }^{\dagger}$ Niclas Luick, Lennart Sobirey, Jonas Siegl, Thomas Lompe, and Henning Moritz \\ Institut für Laserphysik, Universität Hamburg, Luruper Chaussee 149, 22761 Hamburg, Germany
}

(Received 20 April 2017; revised manuscript received 7 November 2017; published 7 February 2018)

\begin{abstract}
We report on the experimental realization of homogeneous two-dimensional (2D) Fermi gases trapped in a box potential. In contrast to harmonically trapped gases, these homogeneous $2 \mathrm{D}$ systems are ideally suited to probe local as well as nonlocal properties of strongly interacting many-body systems. As a first benchmark experiment, we use a local probe to measure the density of a noninteracting 2D Fermi gas as a function of the chemical potential and find excellent agreement with the corresponding equation of state. We then perform matter wave focusing to extract the momentum distribution of the system and directly observe Pauli blocking in a near unity occupation of momentum states. Finally, we measure the momentum distribution of an interacting homogeneous $2 \mathrm{D}$ gas in the crossover between attractively interacting fermions and bosonic dimers.
\end{abstract}

DOI: 10.1103/PhysRevLett.120.060402

Ultracold 2D Fermi gases are uniquely suited to investigate the interplay of reduced dimensionality and strong interactions in quantum many-body systems in a clean and well-controlled environment. Experiments have reported on the creation of 2D Fermi gases with equal $[1,2]$ and unequal spin populations $[3,4]$ and investigated pairing [5-8], Fermi-liquid [9], and polaron physics [10,11]. The equation of state (EOS) [12-14] was determined, and evidence for pair condensation [15] and for a Berezinskii-Kosterlitz-Thouless transition [16] could be observed. Yet so far, ultracold 2D Fermi gases have always been studied in harmonic trapping potentials, which qualitatively change the density of states and give rise to inhomogeneous density distributions. This hinders the observation of critical phenomena with a diverging correlation length and exotic phases such as the Fulde-FerrellLarkin-Ovchinnikov (FFLO) state [17-20]. Furthermore, the inhomogeneous density distribution complicates the interpretation of nonlocal quantities such as correlation functions or momentum distributions, which can be extracted only as trap-averaged quantities $[15,16]$.

These issues can be overcome by creating homogeneous gases in box potentials whose walls are formed by repulsive optical dipole potentials. Following this method, threedimensional (3D) uniform Bose gases have recently been realized and used to investigate coherence and thermodynamic properties $[21,22]$ as well as nonequilibrium dynamics [23]. In homogeneous 2D Bose gases, the emergence of

Published by the American Physical Society under the terms of the Creative Commons Attribution 4.0 International license. Further distribution of this work must maintain attribution to the author(s) and the published article's title, journal citation, and DOI. condensation, vortices, and supercurrents was studied [24,25]. Very recently, the creation of 3D Fermi gases in a box potential has been demonstrated, Pauli blocking in momentum space was observed, and both balanced and imbalanced Fermi gases have been studied in the strongly interacting regime [26].

Here, we report on the experimental realization of homogeneous 2D Fermi gases with tunable interactions. By preparing a noninteracting Fermi gas, we realize a textbook example of statistical physics and directly observe Pauli blocking in the occupation of momentum states.

To measure the momentum distribution of interacting gases, we have established a technique to rapidly remove one spin component and thereby project the system onto a noninteracting state. We apply this technique to a gas with intermediate attractive interactions and observe a momentum distribution that is qualitatively similar to that of a noninteracting gas.

We perform our experiments with an equal spin mixture of ${ }^{6} \mathrm{Li}$ atoms in the lowest two hyperfine states $\left|F, m_{F}\right\rangle=\left|\frac{1}{2}, \frac{1}{2}\right\rangle$ and $\left|\frac{1}{2},-\frac{1}{2}\right\rangle$, which we designate as $|\uparrow\rangle$ and $|\downarrow\rangle$, respectively. A sketch of the experimental setup is shown in Fig. 1(a). The atoms are precooled as described in Ref. [27] and then transferred into a hybrid trap consisting of a highly elliptic red-detuned optical trap and a variable radial magnetic confinement, which is generated by the curvature of the magnetic offset field used to tune the interparticle interactions [28]. This variable trapping can be used to compensate for anticonfinement introduced by the lattice potential, which provides the $2 \mathrm{D}$ confinement described below. After a forced evaporative cooling in the elliptic trap [Fig. 1(d)], we ramp on a repulsive optical ring potential as sketched in Figs. 1(b) and 1(c). This ring potential is generated by a cascaded setup of three axicons and projected onto the atoms using a high-resolution $(\mathrm{NA}=0.62)$ 


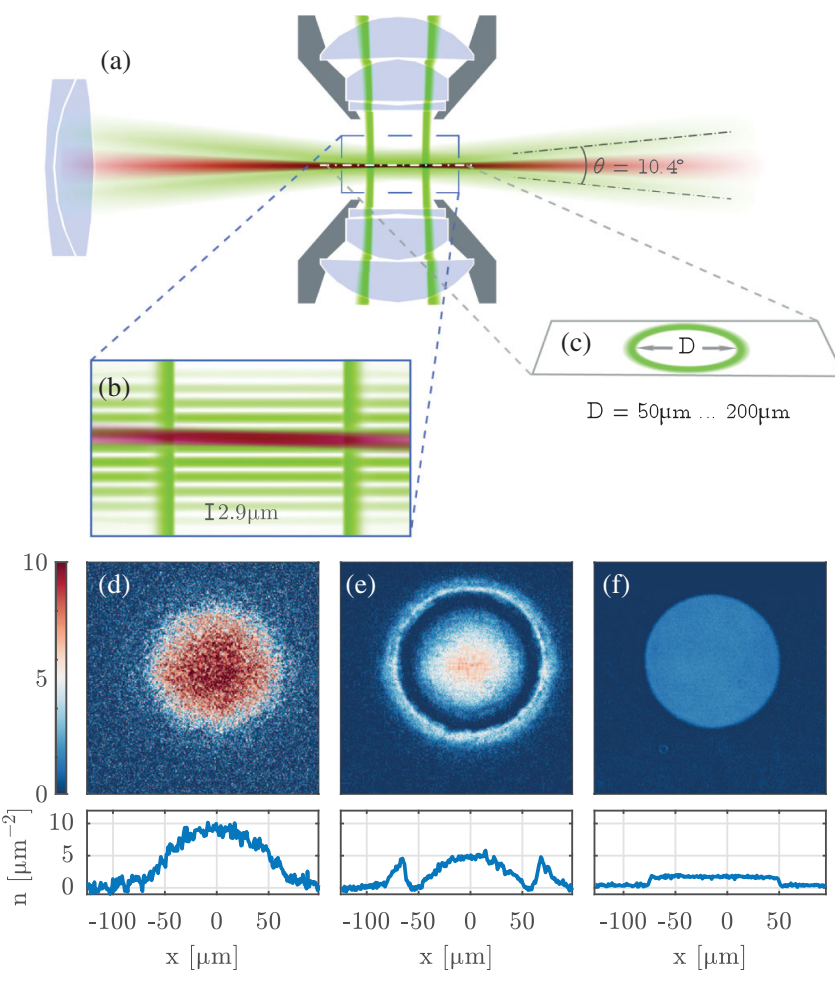

FIG. 1. Sketch of the experimental setup: The atoms are loaded from a highly elliptic red detuned optical trap (red) into a single nodal plane of a blue detuned optical lattice (light green) which is formed by two laser beams $(\lambda=532 \mathrm{~nm})$ intersecting under an opening angle of $\theta=10.4^{\circ}$ (a),(b). The radial confinement is provided by a ring-shaped repulsive potential (dark green) whose diameter $D$ can be adjusted between 50 and $200 \mu \mathrm{m}$ (c). Panels (d)-(f) show averaged (20-50 images) in situ density profiles and the respective central line cuts at different stages of the preparation of a strongly interacting homogeneous Fermi gas at $B=830 \mathrm{G}$ : After evaporation in the elliptic trap (d), the outer, high-entropy region of the cloud is cut away by the repulsive ring potential (e). After further evaporation, the radial magnetic confinement is ramped down to spill the atoms outside the ring, the atoms are transferred into the lattice, and we obtain a homogeneous 2D gas (f).

objective $[26,28,30,31]$. We use the ring to cut out the central, low-entropy part of the cloud [Fig. 1(e)] and then ramp down the radial magnetic confinement such that the excess atoms outside the ring leave the observation volume.

Next, we bring the gas into the $2 \mathrm{D}$ regime by loading it into a blue-detuned optical lattice in the $z$ direction. In this lattice, the level spacing $\hbar \omega_{z}=h(12.4 \pm 0.1) \mathrm{kHz}$ between the ground and the first excited state in the vertical direction exceeds both the highest chemical potential $\mu<h \times 4 \mathrm{kHz}$ and the highest thermal energy $k_{B} T<$ $h \times 2 \mathrm{kHz}$ encountered during our experiments, and hence the system is in the $2 \mathrm{D}$ regime $[32,33]$.

To transfer the atoms into a single node of the lattice, we recompress the cloud by ramping up the power of the elliptic trap, which reduces the width of the cloud in the $z$ direction below the lattice spacing of $2.9 \mu \mathrm{m}$. By optimizing the position of the elliptic trap with respect to the lattice, optimally $93 \%$ of the atoms can be loaded into a single layer, where the number of atoms in adjacent layers can be determined to a high precision in a single-shot matter wave focusing measurement [28]. By shifting the $z$ position of the elliptic trap by half a lattice period, it is also possible to create two equally populated adjacent layers $[28,34]$. This makes the loading of noninteracting gases more robust against populating adjacent layers by thermally excited atoms and furthermore doubles the recorded signal for absorption imaging.

In a first series of experiments, we study a noninteracting Fermi gas, which provides us with a well-defined starting point for our exploration of interacting systems. To create such noninteracting systems, we first prepare a dual-layer homogeneous 2D Fermi gas at a magnetic offset field of $B=320 \mathrm{G}$. At this field, the gas is weakly interacting with a $3 \mathrm{D}$ scattering length of $a_{3 \mathrm{D}}=-290 a_{0}$, where $a_{0}$ is the Bohr radius. We perform further evaporative cooling by slowly decreasing the height of the confining ring potential and then ramp to $B=527 \mathrm{G}$, which is close to the zero crossing of the scattering length, to obtain a noninteracting Fermi gas.

As a first benchmark experiment, we measure the density EOS $n_{2 \mathrm{D}, \uparrow}(\mu, T)$ of this noninteracting Fermi gas. We imprint a potential step, which is generated by a bluedetuned laser beam reflected off the surface of a digital micromirror device (DMD) and projected onto the atoms [35]. We then image the resulting density distribution using high-intensity absorption imaging [28,36,37]. As shown in Figs. 2(a) and 2(b), the repulsive potential causes a diskshaped density depletion in the center of the cloud which covers about $10 \%$ of its area. We apply potential steps with different heights $V$ while observing the corresponding density depletion $\Delta n(V)=n_{2 \mathrm{D}, \uparrow}^{\text {disk }}-n_{2 \mathrm{D}, \uparrow}^{\text {center }}(V)$, where $n_{2 \mathrm{D}, \uparrow}^{\text {disk }}$ and $n_{2 \mathrm{D} . \uparrow}^{\text {certer }}$ correspond to the single-layer density in the undisturbed and depleted parts of the trap, respectively. We perform such EOS measurements for gases with different densities and temperatures; the resulting data sets are shown in Fig. 2.

We calibrate the potential step height $V$ by performing a linear Thomas-Fermi fit to the first four points of the different EOS measurements and take the mean of the resulting values [14]. To extract the temperature and chemical potential, we fit the density depletion with $\Delta n\left(\mu_{0}, T, V\right)=n_{2 \mathrm{D}, \uparrow}\left(\mu_{0}, T\right)-n_{2 \mathrm{D}, \uparrow}\left(\mu_{0}-V, T\right)$ using the theoretical $\operatorname{EOS} n_{2 \mathrm{D}, \uparrow}(\mu, T)=\lambda_{\mathrm{dB}}^{-2} \log [1+\exp (\mu \beta)]$ for a noninteracting 2D Fermi gas [38]. Here, $\beta=\left(k_{B} T\right)^{-1}$, and the thermal de Broglie wavelength is given by $\lambda_{\mathrm{dB}}=\sqrt{2 \pi \hbar^{2} / m k_{B} T}$, where $m$ is the mass of a ${ }^{6} \mathrm{Li}$ atom. We approximate the chemical potential $\mu_{0}$ in the outer part of the trap to be constant for all step heights. For our coldest data set, we obtain a temperature of $T / T_{F}=0.14 \pm 0.02$, where the Fermi temperature $T_{F}$ is calculated from $T$ and $\mu_{0}$ using $T_{F}=T \log \left[1+\exp \left(\beta \mu_{0}\right)\right]$ [39].

We validate these measurements by plotting the dimensionless quantity $n_{2 \mathrm{D}, \uparrow} \lambda_{\mathrm{dB}}^{2}$ as a function of $\beta \mu$ for each of the 




FIG. 2. Density EOS for noninteracting homogeneous 2D Fermi gases: The EOS is mapped out for different densities and temperatures by imprinting a repulsive potential step onto the atoms. This causes a density depletion $\Delta n$ in the center of the cloud (a),(b). Measuring this density depletion $\Delta n$ as a function of the step height directly yields the density EOS of the system. By fitting the data with the EOS of a noninteracting Fermi gas, we extract the temperature $T$ and chemical potential $\mu_{0}$ for each data set. The higher $T / T_{F}$ for the data set having the lowest density in the outer ring (red squares) is most likely due to a reduced evaporation efficiency. Using the fit results for $T$ and $\mu$ to rescale the data and plotting the dimensionless quantity $n_{2 \mathrm{D}, \uparrow} \lambda_{\mathrm{dB}}^{2}$ causes the different data sets to collapse onto a single curve (c). The data show excellent agreement with the prediction for a noninteracting 2D Fermi gas (solid purple line).

different systems [Fig. 2(c)] [40]. The different data sets all collapse onto a single curve and are in excellent agreement with the theoretical expectation.

We now go beyond this local probing of the density and chemical potential by performing a direct measurement of the momentum distribution of an ideal 2D Fermi gas. We achieve this by mapping the momentum distribution to real space using matter wave focusing [15,41-43]: We switch off the radial confinement provided by the ring potential and let the system evolve for a time $t$ in a weak harmonic potential in the radial direction. After a time evolution of a quarter of the radial trap period $\tau=2 \pi / \omega_{r}$, all particles with momentum $\hbar \mathbf{k}$ have moved to a position $\mathbf{r}=\hbar \mathbf{k} / m \omega_{r}$. Hence, the momentum distribution $\tilde{n}(\mathbf{k})$ can be directly extracted from the density distribution $n(\mathbf{r}, t)$ at $t=\tau / 4$ via $\tilde{n}(\mathbf{k})=$ $\left(\hbar / m \omega_{r}\right)^{2} n\left(\mathbf{r}=\hbar \mathbf{k} / m \omega_{r}, \tau / 4\right)$ [Figs. 3(b), 3(e), and 3(h)].

This technique can also be extended to perform matter wave imaging instead of matter wave focusing by letting the system evolve for $t=\tau / 2$ instead of $t=\tau / 4$ [44]. This causes the initial density distribution to reappear inverted around the center of the trap, i.e., $n(\mathbf{r}, \tau / 2)=n(-\mathbf{r}, 0)$. Comparing the matter wave imaged distribution at $t=\tau / 2$ with the initial distribution provides a measure for the quality of the matter wave lens formed by the radial potential, which can be affected by anharmonicities of the
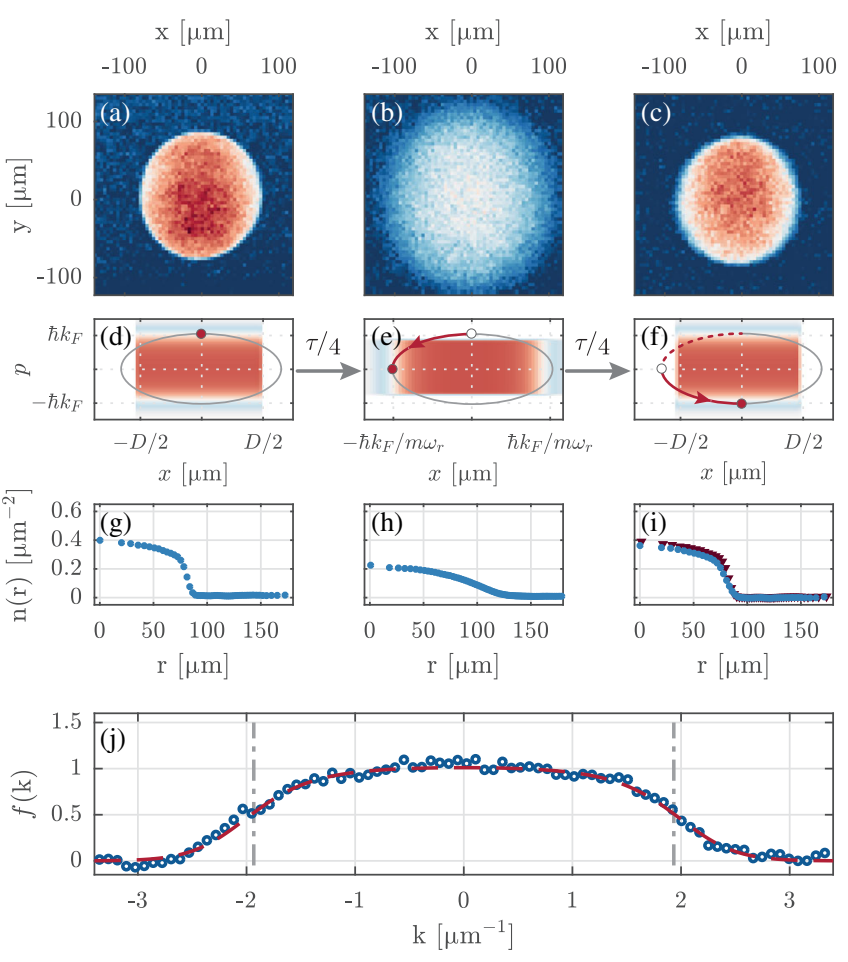

FIG. 3. Momentum distribution of a noninteracting 2D Fermi gas: To measure the momentum distribution, we switch off the confining ring potential and let the gas evolve in a weak harmonic potential. A free time evolution $t$ for a quarter of the trap period $\tau$ performs a rotation in phase space by $90^{\circ}$ as sketched in (d),(e), causing the momentum distribution of the gas to be mapped into real space. Averaged images (51-62 realizations) and corresponding azimuthal averages of the density and momentum distribution are shown in (a),(b) and $(\mathrm{g}),(\mathrm{h})$, respectively. After a free time evolution of half a trap period, the in situ density distribution is mapped back to real space (c); the azimuthal averages at $t=0$ (red triangles) and $t=\tau / 2$ (blue dots) are almost identical (i). A diagonal cut through the momentum distribution (b) reveals the occupation $f(k)$ of the system (j), which shows close to unity occupation around $k=0$ and drops off at the Fermi wave vector $k_{F}=(1.93 \pm 0.02) \mu \mathrm{m}^{-1} \quad$ (gray dash-dotted lines). A fit with a Fermi distribution (red dashed line) yields a temperature of $T / T_{F}=0.31 \pm 0.02$.

potential. For our experiments, we set the radial magnetic trap frequency to a value of $\omega_{r}=2 \pi(33.3 \pm 0.5) \mathrm{Hz}$ and ramp down the depth of the $z$ confinement by a factor of 5 to minimize the influence of its antitrapping potential while keeping the atoms in the depth of field. We find that the in situ and matter wave imaged density distributions are virtually indistinguishable [Figs. 3(a), 3(c), and 3(i)], which shows that, for this noninteracting system, our matter wave focusing gives an accurate measurement of the momentum distribution.

To extract the occupation $f(\mathbf{k})=A_{k} \tilde{n}(\mathbf{k})$ from the momentum distribution $\tilde{n}(\mathbf{k})$, we use the $k$-space area $A_{k}=$ $16 \pi / \mathrm{D}^{2}$ of a single $k$ mode in a box potential with diameter $D$. This allows us to directly observe Pauli blocking in our 
noninteracting Fermi gas, which manifests itself in a unity occupation of $k$ modes around $k=0$, followed by a drop in the occupation at the Fermi wave vector $k_{F}$ [Fig. 3(j)].

Next, we quantitatively determine the chemical potential and the temperature of the gas by fitting our data with the Fermi distribution

$$
f(k)=\frac{\zeta}{1+\exp \left[\beta\left(\frac{\hbar^{2} k^{2}}{2 m}-\mu_{0}\right)\right]} .
$$

The free parameters of the fit are the temperature $T$, the chemical potential $\mu_{0}$, and an overall amplitude $\zeta$ which accounts for systematic errors in the determination of $\tilde{n}(\mathbf{k})$ and $A_{k}$. The fit is in excellent agreement with the data [Fig. 3(j)] and yields a chemical potential $\mu_{0}=$ $k_{B}(148.8 \pm 2.6) \mathrm{nK}$, a temperature $T=(46.7 \pm 2.2) \mathrm{nK}$, and $\zeta=1.05 \pm 0.06$, where the errors denote $1 \sigma$ confidence intervals of the fit. The dominant sources of systematic errors on the amplitude of $f(k)$ are the $2 \%$ uncertainty of the radial trap frequency $\omega_{r}$, the $7 \%$ uncertainty in the density calibration, and the $4 \%$ uncertainty in the determination of the ring diameter $D$ from the in situ images. The fit results translate to $T / T_{F}=0.31 \pm 0.02, \quad \mu_{0} / \hbar \omega_{z}=0.250 \pm 0.005, \quad$ and $\mathrm{a}$ Fermi wave vector $k_{F}=(1.93 \pm 0.02) \mu \mathrm{m}^{-1}$. This is in very good agreement with both the Fermi wave vector deduced from the mean density $k_{F, \bar{n}}=\sqrt{4 \pi \bar{n}_{2 \mathrm{D}, \uparrow}}=$ $(1.86 \pm 0.08) \mu \mathrm{m}^{-1}$ and the temperature and chemical potential obtained for a similar evaporation depth in the EOS measurement shown in Fig. 2 (red solid line) [45]. We note that the fitted temperature is an upper bound, affected by fluctuations in the particle number and the inhomogeneity of the density distribution, which is smaller than $11 \%$ of the mean density [28]. This value includes both the actual density inhomogeneity due to the presence of the harmonic potential used for the matter wave focusing and artifacts due to imperfections of the imaging beam.

When measuring the momentum distribution for varying densities [see Fig. 4(a)], we observe that the occupation at low momenta saturates to values close to unity for densities ranging from 0.25 to $0.5 \mu \mathrm{m}^{-2}$. This clearly shows Pauli blocking in momentum space [26].

Finally, we realize an interacting homogeneous 2D Fermi gas close to a broad Feshbach resonance [46] and apply matter wave focusing. We prepare a single-layer attractive 2D Fermi gas at $B=1020 \mathrm{G}$ where the ratio of scattering length $a_{3 \mathrm{D}}$ to harmonic oscillator length $l_{z}=$ $\sqrt{\hbar / m \omega_{z}}$ is $a_{3 \mathrm{D}} / l_{z}=-0.56$. In contrast to previous experiments which measured the pair momentum distribution by converting pairs into deeply bound molecules [15], we measure the momentum distribution of the individual atoms [47]. This requires a negligible influence of collisions on the time evolution. We achieve this by releasing the gas from the vertical confinement $[48,49]$ as well as
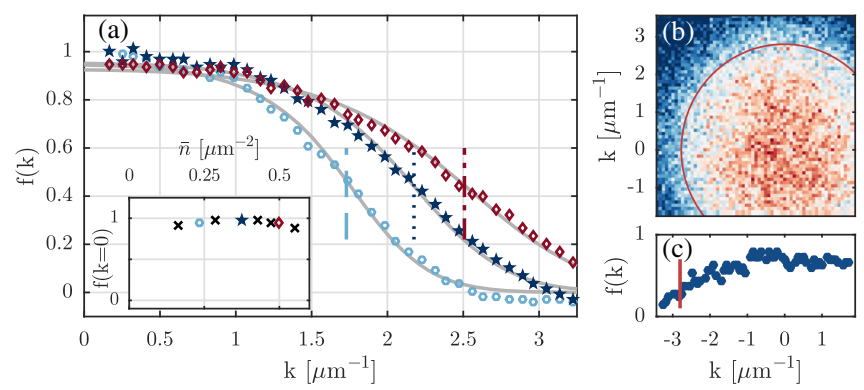

FIG. 4. Saturation in the occupation of momentum states. The occupation $f(k)$ of noninteracting Fermi gases with in situ densities of $\bar{n}=0.24$ (light blue hexagons), 0.38 (dark blue stars), and $0.50 \mu \mathrm{m}^{-2}$ (red diamonds) is shown in (a). For low momenta, we find a near unity occupation that is independent of the in situ density (see the inset), which is direct evidence of Pauli blocking. The Fermi wave vectors deduced from Fermi fits to the distribution agree well with the $k_{F, \bar{n}}=\sqrt{4 \pi \bar{n}_{2 \mathrm{D}, \uparrow}}$ (vertical lines) calculated from the in situ density. An image of the momentum distribution of an attractively interacting Fermi gas is shown in panel (b) and a cut through the distribution in panel (c). The Fermi momentum $k_{F, \bar{n}}$ for a noninteracting gas with equal density is indicated by the red circle (b) and the vertical red line (c), respectively.

flashing on a light pulse propagating along the $z$ direction which rapidly ejects atoms in state $|\uparrow\rangle[26,28,50,51]$. This projects the wave function of atoms in state $|\downarrow\rangle$ onto free particle states and allows us to extract the occupation $f(k)$ of the interacting system using the matter wave focusing technique described above. For our interaction strength, we expect only small deviations in $f(k)$ compared to the noninteracting system, since at $T=0$ the quasiparticle weight $Z$ and the gap $\Delta$ are calculated to be $Z \approx 0.9$ [52] and $\Delta \approx 21 \%$ [53]. We therefore attribute the reduced central occupation and the broadening of the momentum distribution shown in Fig. 4(e) to thermal excitations.

In this Letter, we report on the realization of a homogeneous 2D Fermi gas trapped in a box potential. We locally probe the system by imprinting a step potential using a DMD and thereby measure the EOS of a noninteracting Fermi gas. Furthermore, we apply matter wave focusing to directly observe Pauli blocking in the momentum distribution of a noninteracting 2D Fermi gas. Finally, we demonstrate that the momentum distribution of interacting gases can also be measured and observe a momentum distribution that is qualitatively similar to that of a noninteracting gas for intermediate interactions.

The homogeneous systems presented in this work are particularly useful for studying nonequilibrium dynamics of strongly correlated systems, since they allow interaction quenches without triggering a mass redistribution, which is unavoidable in harmonic traps. The combination of such a homogeneous system with nonlocal probes is ideally suited to observe critical phenomena and exotic phases such as FFLO superfluidity, which are predicted to exist only in narrow regions of the phase diagram. Finally, our 
measurement of the momentum distribution of an interacting Fermi gas can be extended to analyze momentum correlations [54] and thereby observe Cooper pairs in a fermionic superfluid.

We thank W. Weimer, K. Morgener, and K. Riechers for their contributions during earlier stages of the experiment and F. Werner and M. W. Zwierlein for their helpful comments. This work was supported by the European Union's Seventh Framework Program (FP7/2007-2013) under Grant Agreement No. 335431 and by the DFG in the framework of SFB 925, GrK 1355 and the excellence cluster "The Hamburg Centre for Ultrafast Imaging".

*Corresponding author.

henning.moritz@physik.uni-hamburg.de

${ }^{\dagger}$ khueck@physik.uni-hamburg.de

[1] K. Martiyanov, V. Makhalov, and A. Turlapov, Phys. Rev. Lett. 105, 030404 (2010).

[2] P. Dyke, E. D. Kuhnle, S. Whitlock, H. Hu, M. Mark, S. Hoinka, M. Lingham, P. Hannaford, and C. J. Vale, Phys. Rev. Lett. 106, 105304 (2011).

[3] W. Ong, C. Cheng, I. Arakelyan, and J. E. Thomas, Phys. Rev. Lett. 114, 110403 (2015).

[4] D. Mitra, P. T. Brown, P. Schauß, S. S. Kondov, and W. S. Bakr, Phys. Rev. Lett. 117, 093601 (2016).

[5] B. Fröhlich, M. Feld, E. Vogt, M. Koschorreck, W. Zwerger, and M. Köhl, Phys. Rev. Lett. 106, 105301 (2011).

[6] M. Feld, B. Fröhlich, E. Vogt, M. Koschorreck, and M. Köhl, Nature (London) 480, 75 (2011).

[7] A. T. Sommer, L. W. Cheuk, M. J. H. Ku, W. S. Bakr, and M. W. Zwierlein, Phys. Rev. Lett. 108, 045302 (2012).

[8] C. Cheng, J. Kangara, I. Arakelyan, and J. E. Thomas, Phys. Rev. A 94, 031606(R) (2016).

[9] B. Fröhlich, M. Feld, E. Vogt, M. Koschorreck, M. Köhl, C. Berthod, and T. Giamarchi, Phys. Rev. Lett. 109, 130403 (2012).

[10] M. Koschorreck, D. Pertot, E. Vogt, B. Fröhlich, M. Feld, and M. Köhl, Nature (London) 485, 619 (2012).

[11] Y. Zhang, W. Ong, I. Arakelyan, and J. E. Thomas, Phys. Rev. Lett. 108, 235302 (2012).

[12] V. Makhalov, K. Martiyanov, and A. Turlapov, Phys. Rev. Lett. 112, 045301 (2014).

[13] K. Fenech, P. Dyke, T. Peppler, M. G. Lingham, S. Hoinka, H. Hu, and C. J. Vale, Phys. Rev. Lett. 116, 045302 (2016).

[14] I. Boettcher, L. Bayha, D. Kedar, P. A. Murthy, M. Neidig, M. G. Ries, A. N. Wenz, G. Zürn, S. Jochim, and T. Enss, Phys. Rev. Lett. 116, 045303 (2016).

[15] M. G. Ries, A. N. Wenz, G. Zürn, L. Bayha, I. Boettcher, D. Kedar, P. A. Murthy, M. Neidig, T. Lompe, and S. Jochim, Phys. Rev. Lett. 114, 230401 (2015).

[16] P. A. Murthy, I. Boettcher, L. Bayha, M. Holzmann, D. Kedar, M. Neidig, M. G. Ries, A. N. Wenz, G. Zürn, and S. Jochim, Phys. Rev. Lett. 115, 010401 (2015).

[17] P. Fulde and R. A. Ferrell, Phys. Rev. 135, A550 (1964).

[18] A. Larkin and Y. N. Ovchinnikov, Sov. Phys. JETP 20, 762 (1965).
[19] G. J. Conduit, P. H. Conlon, and B. D. Simons, Phys. Rev. A 77, 053617 (2008).

[20] U. Toniolo, B. Mulkerin, X.-J. Liu, and H. Hu, Phys. Rev. A 95, 013603 (2017).

[21] A. L. Gaunt, T. F. Schmidutz, I. Gotlibovych, R. P. Smith, and Z. Hadzibabic, Phys. Rev. Lett. 110, 200406 (2013).

[22] T. F. Schmidutz, I. Gotlibovych, A. L. Gaunt, R. P. Smith, N. Navon, and Z. Hadzibabic, Phys. Rev. Lett. 112, 040403 (2014).

[23] A. L. Navon, N. Gaunt, R. P. Smith, and Z. Hadzibabic, Science 347, 167 (2015).

[24] L. Corman, L. Chomaz, T. Bienaimé, R. Desbuquois, C. Weitenberg, S. Nascimbène, J. Dalibard, and J. Beugnon, Phys. Rev. Lett. 113, 135302 (2014).

[25] L. Chomaz, L. Corman, T. Bienaimé, R. Desbuquois, C. Weitenberg, S. Nascimbène, J. Beugnon, and J. Dalibard, Nat. Commun. 6, 6162 (2015).

[26] B. Mukherjee, Z. Yan, P. B. Patel, Z. Hadzibabic, T. Yefsah, J. Struck, and M. W. Zwierlein, Phys. Rev. Lett. 118, 123401 (2017).

[27] W. Weimer, K. Morgener, V. P. Singh, J. Siegl, K. Hueck, N. Luick, L. Mathey, and H. Moritz, Phys. Rev. Lett. 114, 095301 (2015).

[28] See Supplemental Material at http://link.aps.org/ supplemental/10.1103/PhysRevLett.120.060402 for information about optical trap geometries and loading procedures, the density determination and the matter wave focusing of interacting gases, which includes Ref. [29].

[29] M. Pappa, P. C. Condylis, G. O. Konstantinidis, V. Bolpasi, A. Lazoudis, O. Morizot, D. Sahagun, M. Baker, and W. von Klitzing, New J. Phys. 13, 115012 (2011).

[30] J. H. McLeod, J. Opt. Soc. Am. 44, 592 (1954).

[31] I. Manek, Y. Ovchinnikov, and R. Grimm, Opt. Commun. 147, 67 (1998).

[32] D. S. Petrov, M. Holzmann, and G. V. Shlyapnikov, Phys. Rev. Lett. 84, 2551 (2000).

[33] P. Dyke, K. Fenech, T. Peppler, M. G. Lingham, S. Hoinka, W. Zhang, S.-G. Peng, B. Mulkerin, H. Hu, X.-J. Liu, and C. J. Vale, Phys. Rev. A 93, 011603 (2016).

[34] Z. Hadzibabic, P. Krüger, M. Cheneau, B. Battelier, and J. Dalibard, Nature (London) 441, 1118 (2006).

[35] K. Hueck, A. Mazurenko, N. Luick, T. Lompe, and H. Moritz, Rev. Sci. Instrum. 88, 016103 (2017).

[36] G. Reinaudi, T. Lahaye, Z. Wang, and D. Guéry-Odelin, Opt. Lett. 32, 3143 (2007).

[37] K. Hueck, N. Luick, L. Sobirey, T. Lompe, H. Moritz, L. Clark, and C. Chin, Opt. Express 25, 8670 (2017).

[38] M. Bauer, M. M. Parish, and T. Enss, Phys. Rev. Lett. 112, 135302 (2014).

[39] This equation is found by solving the 2D EOS for $\mu_{0}$, taking the $T=0$ limit, yielding $T_{F}=n_{2 \mathrm{D}, \uparrow} 2 \pi \hbar^{2} /\left(m k_{B}\right)$, and reinserting the EOS for $n_{2 \mathrm{D}, \uparrow}$.

[40] C.-L. Hung, X. Zhang, N. Gemelke, and C. Chin, Nature (London) 470, 236 (2011).

[41] I. Shvarchuck, C. Buggle, D. S. Petrov, K. Dieckmann, M. Zielonkovski, M. Kemmann, T. G. Tiecke, W. von Klitzing, G. V. Shlyapnikov, and J. T. M. Walraven, Phys. Rev. Lett. 89, 270404 (2002).

[42] S. Tung, G. Lamporesi, D. Lobser, L. Xia, and E. A. Cornell, Phys. Rev. Lett. 105, 230408 (2010). 
[43] P. A. Murthy, D. Kedar, T. Lompe, M. Neidig, M. G. Ries, A. N. Wenz, G. Zürn, and S. Jochim, Phys. Rev. A 90, 043611 (2014).

[44] In our case, this imaging has a magnification of 1 . Other magnifications are accessible by switching to a different radial trap frequency after the $\tau / 4$ point.

[45] We chose a low evaporation depth, despite the fact that we achieve our lowest $T / T_{F}$ at higher Fermi energies, since we want $k_{F}$ to be small enough that the full momentum distribution is captured by the field of view of the imaging system.

[46] G. Zürn, T. Lompe, A. N. Wenz, S. Jochim, P. S. Julienne, and J. M. Hutson, Phys. Rev. Lett. 110, 135301 (2013).

[47] C. A. Regal, M. Greiner, S. Giorgini, M. Holland, and D. S. Jin, Phys. Rev. Lett. 95, 250404 (2005).
[48] P. Krüger, Z. Hadzibabic, and J. Dalibard, Phys. Rev. Lett. 99, 040402 (2007).

[49] P. Cladé, C. Ryu, A. Ramanathan, K. Helmerson, and W. D. Phillips, Phys. Rev. Lett. 102, 170401 (2009).

[50] M. Weinberg, O. Jürgensen, C. Ölschläger, D.-S. Lühmann, K. Sengstock, and J. Simonet, Phys. Rev. A 93, 033625 (2016).

[51] S. Murmann, F. Deuretzbacher, G. Zürn, J. Bjerlin, S. M. Reimann, L. Santos, T. Lompe, and S. Jochim, Phys. Rev. Lett. 115, 215301 (2015).

[52] P. Bloom, Phys. Rev. B 12, 125 (1975).

[53] A. M. Fischer and M. M. Parish, Phys. Rev. B 90, 214503 (2014).

[54] M. Greiner, C. A. Regal, J. T. Stewart, and D. S. Jin, Phys. Rev. Lett. 94, 110401 (2005). 